\title{
VARIACIONES INTRATALINAS DE LAS SUSTANCIAS LIQUÉNICAS CON LA EDAD EN Lasallia PUStulata (L.) MÉrat.
}

\author{
Estela SERIÑÁ, Rosario ARROYO \& Esteban MANRIQUE
}

\begin{abstract}
RESUMEN: Utilizando la cromatografía líquida de alta resolución, se estudia la distribución de sustancias liquénicas a través del talo de Lasallia pustulata (L.) Mérat y la variación de la concentración de dichas sustancias a lo largo de la vida del liquen. Los ácidos lecanórico y girofórico se acumulan más, por unidad de peso seco, en la periferia del talo y se presentan en mayor proporción en los talos más jóvenes.
\end{abstract}

Palabras clave: Líquenes, sustancias liquénicas, variación intratalina.

ABSTRACT: The distribution of lichen substances within the tallus of Lasallia pustulata (L.) Mérat, and the variation in concentration of these substances with thallus age was studied using high performance liquid chromatography. Lecanoric and gyrophoric acids were presents in greatest quantity per unit weigh at the thallus periphery and also in the youngest thalli studied.

Key words: Lichens, lichen substances, intrathalline variation.

\section{INTRODUCCIÓN}

Hasta el momento son pocos los trabajos existentes respecto a la distribución intratalina de las sustancias liquénicas. La presencia de dichas sustancias parece ser constante desde el punto de vista cualitativo, independientemente de la edad o el tamaño del liquen (Culberson \& Culberson, 1958). No obstante, muy poco se conoce acerca de si su concentración puede verse modificada según las diferentes zonas del talo que se analicen o si la proporción de los ácidos liquénicos con relación al tamaño del liquen sufre variaciones en las sucesivas etapas de crecimiento (Fahselt, 1984; Geyer, 1985; López Redondo, 1988).

Para profundizar en estos aspectos, objetivo del presente trabajo, se ha elegido Lasallia pustulata (L.) Mérat por ofrecer unas características morfológicas y de forma de crecimiento ideales para poder estudiar la distribución de sustancias radialmente y también en una serie de individuos en diferentes estados de desarrollo. 


\section{MATERIAL Y MÉTODOS}

Se han seleccionado 30 especímenes de una misma localidad (España, Madrid, Miraflores de la Sierra, Puerto de la Morcuera, Arroyo de Santa Ana, 1500 m.s.m., 30TVL23, sobre gneis, MAF), sobre la misma roca y con idéntica exposición, a partir de una única población para asegurar así la homogeneidad genética de la misma.

La diferencia de edad se puso de manifiesto por diferencias en el tamaño, que se midió por el peso seco al aire de cada ejemplar. Se delimitaron zonas en el talo utilizando dos ejemplares de aproximadamente $6 \mathrm{~cm}$ de diámetro, de los que se extrajeron anillos concéntricos de unos $0.5 \mathrm{~cm}$ de anchura, tomando como primera porción el área ocupada por el ombligo (Fig. 1). Estas porciones y los talos de diferente tamaño se sumergieron en acetona en una proporción de $10 \mu \mathrm{l} / \mathrm{mg}$ peso seco, durante 10 minutos a temperatura ambiente. Los extractos se analizaron por cromatografía en capa fina (TLC) de acuerdo con Culberson \& Kristinsson (1970) y Culberson (1972, 1974) y por cromatografía líquida de alta resolución (HPLC). En este caso se utilizó un Cromatógrafo Perkin Elmer Series 10 equipado con una columna analítica Spherisorb S5 ODS $25 \mu \mathrm{m}$ de $25 \mathrm{~cm}$ x $4.6 \mathrm{~mm}$; la fase móvil es una mezcla de metanolagua-ácido acético (75:25:1). La detección se realizó con un espectrofotómetro Lambda 1 fijado a $270 \mathrm{~nm}$. Los cálculos se realizaron en un registrador-integrador LCI 100 Perkin Elmer. El flujo del disolvente fue de $1 \mathrm{ml} / \mathrm{min}$ (1200 psi) y el volumen de inyección constante $(6 \mu \mathrm{l})$. Los datos, una vez realizados los análisis, se expresaron en unidades arbitrarias de área de cada pico por miligramo de peso seco.
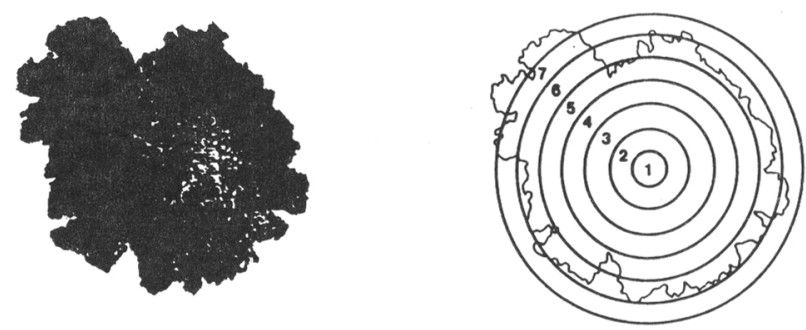

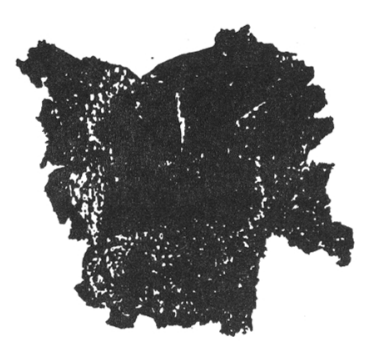

A

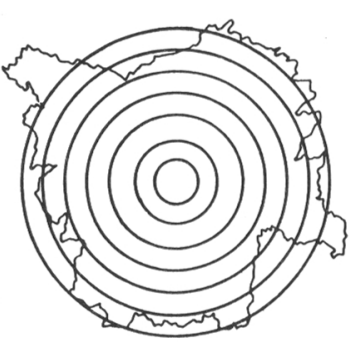

B

Fig. 1. A: Fotocopia de los talos elegidos para el estudio (tamaño real); B: Esquema de los talos y de los anillos analizados de $0.5 \mathrm{~cm}$ de anchura cada uno. 


\section{RESULTADOS Y DISCUSIÓN}

En todos los especímenes analizados por TLC se han detectado como mayoritarios los ácidos lecanórico y girofórico. Los análisis por HPLC (Fig. 2) permiten ver tres picos, dos de ellos asimilables a los ácidos mencionados ( $\mathrm{tR}=5.67$ min, ácido lecanórico y $\mathrm{tR}=11.29 \mathrm{~min}$, ácido girofórico) y el tercero correspondiente a una sustancia no identificada $(\mathrm{tR}=7.26 \mathrm{~min})$ que no se detectó por TLC.

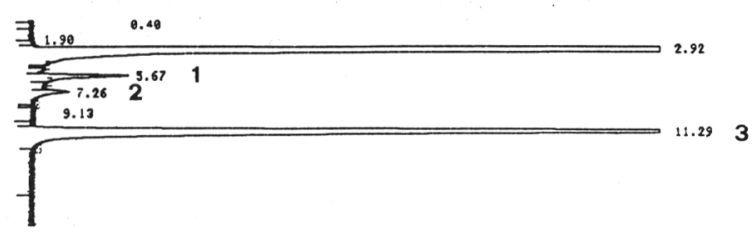

Fig. 2. Cromatograma representativo de los análisis realizados por HPLC en Lasallia pustulata. $1=$ ácido lecanórico; 2 = pico no identificado; 3 = ácido girofórico.

En la Tabla 1 se reflejan las áreas de los picos por $\mathrm{mg}$ de peso seco de las sustancias encontradas en los talos de diferentes pesos analizados. Si se representan gráficamente estos valores para cada uno de los ácidos, en ordenadas área de pico/mg peso seco de cada sustancia y en abscisas peso seco del talo en mg (Fig. 3), se observa como la concentración por unidad de peso de cada una de las sustancias disminuye a medida que aumenta el tamaño del liquen (peso).

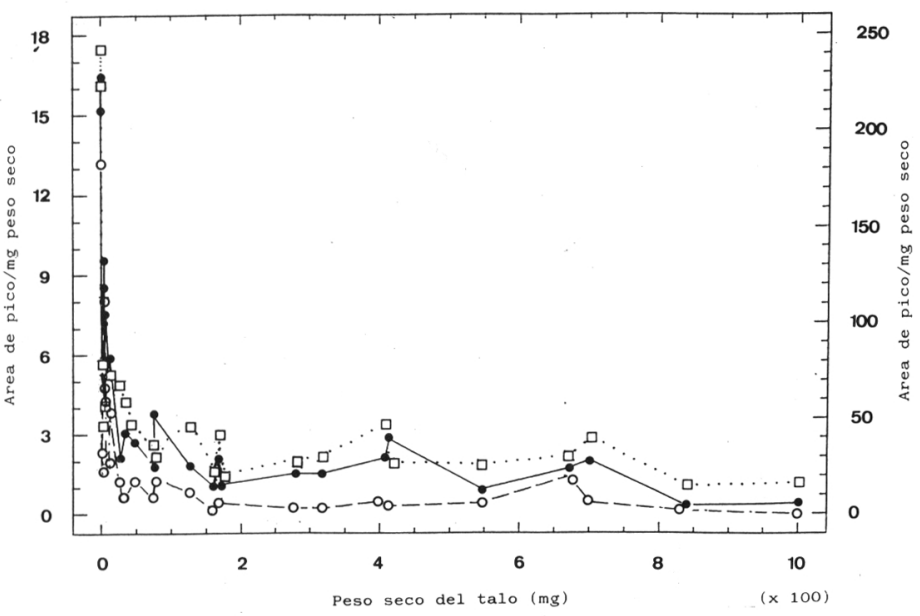

Fig. 3. Representación gráfica de las concentraciones de las tres principales sustancias detectadas en individuos de diferentes tamaños, edades, de Lasallia pustulata. La concentración de cada sustancia expresada en unidades de área de pico por $\mathrm{mg}-1$ peso seco decrece a medida que aumenta el tamaño del liquen (peso). - - - ácido lecanórico; —o- sustancia no identificada; …ㅁ..... ácido girofórico. 


\begin{tabular}{|c|c|c|c|}
\hline Peso seco mg & Area Lec/mg & Area SNI/mg & Area Gyr/mg \\
\hline 0.3 & 15.2 & 13.1 & 224.2 \\
\hline 0.4 & 16.2 & 2.3 & 243.4 \\
\hline 0.9 & 4.2 & 1.7 & 46.7 \\
\hline 1.5 & 8.6 & 1.8 & 78.6 \\
\hline 3.8 & 7.6 & 8.2 & 63.1 \\
\hline 4.5 & 8.5 & 4.1 & 66.9 \\
\hline 4.9 & 5.9 & 5.9 & 71.6 \\
\hline 7.0 & 7.5 & 4.3 & 64.8 \\
\hline 7.3 & 7.2 & 1.9 & 68.7 \\
\hline 11.4 & 5.1 & 1.9 & 65.7 \\
\hline 12.5 & 5.8 & 3.8 & 73.9 \\
\hline 27.6 & 2.1 & 0.8 & 68.1 \\
\hline 34.2 & 3.1 & 0.6 & 58.7 \\
\hline 47.8 & 2.7 & 1.3 & 45.3 \\
\hline 76.7 & 1.8 & 0.5 & 30.2 \\
\hline 74.5 & 3.7 & 1.4 & 37.1 \\
\hline 127.8 & 1.8 & 0.8 & 46.6 \\
\hline 161.5 & 1.0 & 0.1 & 23.1 \\
\hline 166.9 & 2.0 & 0.4 & 42.3 \\
\hline 170.8 & 1.1 & 0.4 & 20.7 \\
\hline 278.3 & 1.5 & 0.2 & 28.2 \\
\hline 316.3 & 1.5 & 0.2 & 30.3 \\
\hline 407.8 & 2.1 & 0.5 & 47.5 \\
\hline 413.9 & 2.8 & 0.3 & 27.1 \\
\hline 546.2 & 0.9 & 0.4 & 26.1 \\
\hline 670.8 & 1.7 & 1.4 & 30.4 \\
\hline 700.7 & 2.0 & 0.4 & 39.7 \\
\hline 837.4 & 0.3 & 0.1 & 14.9 \\
\hline 999.5 & 0.4 & 0.0 & 16.9 \\
\hline
\end{tabular}

Tabla 1. Concentración (Area de pico/mg peso seco) de las sustancias liquénicas detectadas en talos de Lasallia pustulata de diferentes edades (Peso). Lec $=$ Acido lecanórico; SNI= Sustancia no identificada; $\mathrm{Gyr}=$ Acido girofórico. La concentración de las distintas sustancias se expresa en unidades arbitrarias de área de pico/mg peso seco.

Se podría deducir, por tanto, que la síntesis de sustancias liquénicas en esta especie no se realiza por igual a lo largo de la vida del liquen. Tendría lugar con una alta tasa al principio, cuando los talos son todavía muy jóvenes, reteniéndose a medida que éstos aumentan de tamaño.

No obstante, podría ocurrir que dicha tasa de síntesis permaneciera constante y elevada en algunas partes del talo, mientras que en otras podría desaparecer o casi desaparecer. Con el fin de comprobar esta hipótesis se estudió la distribución intratalina de las sustancias en anillos concéntricos. Para ello, se analizaron por HPLC cada uno de los anillos en los dos talos seleccionados, siendo los resultados los valores 
medios de ambos (Tabla 2). La concentración del ácido lecanórico por unidad de peso seco, crece desde el ombligo hacia la periferia. Para el caso del ácido girofórico se observa una tendencia parecida, aunque muestra un máximo de concentración en la zona inmediata al ombligo (Fig. 4). El análisis de regresión para la concentración intratalina del ácido lecanórico en Lasallia pustulata muestra una variación exponencial entre la variación de la concentración del ácido lecanórico y la distancia al centro del talo (Fig. 5).

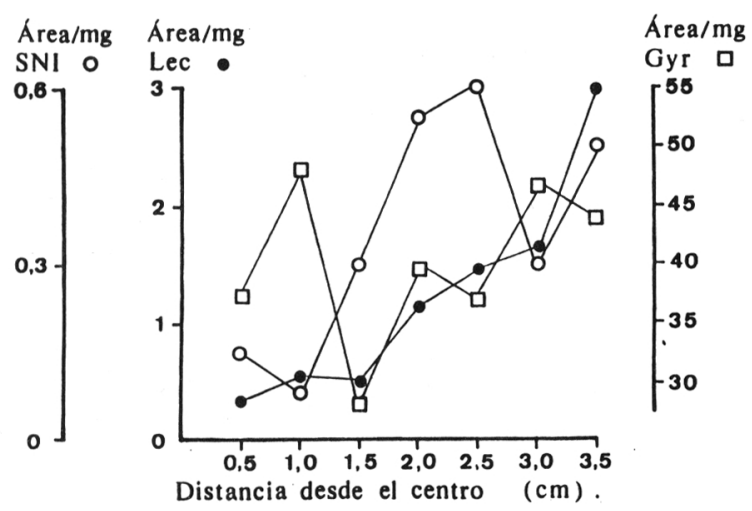

Fig. 4. Variación de las concentraciones de ácido lecanórico, ácido girofórico y SNI a través del talo umbilicado de Lasallia pustulata.

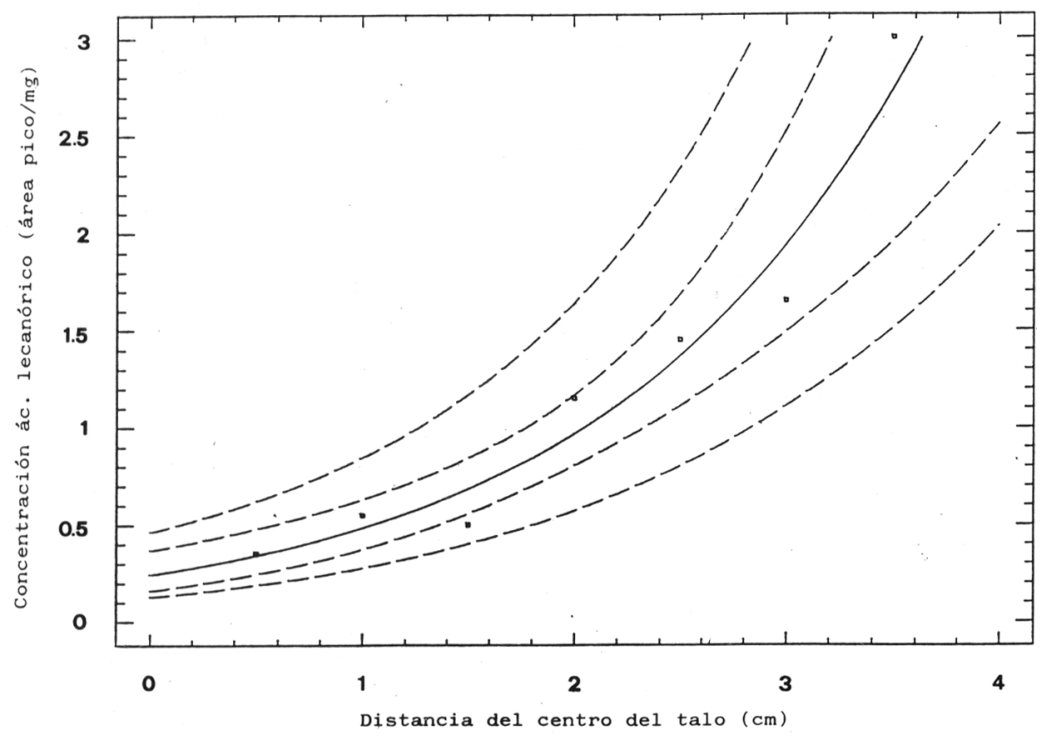

Fig. 5. Análisis de regresión para la concentración intratalina de ácido lecanórico en Lasallia pustulata. Esta variación se aproxima a una exponencial $\mathrm{y}=\mathrm{e}(\mathrm{a}+\mathrm{b}+\mathrm{x})$ con un coeficiente de correlación de 0.97 y un R2 $=94.86 \%$. 


\begin{tabular}{cccc}
\hline № de anillo & Area Lec/mg & Area SNI/ mg & Area Gyr/mg \\
\hline 1 & 0.35 & 0.15 & 37.20 \\
2 & 0.55 & 0.08 & 48.20 \\
3 & 0.50 & 0.30 & 28.10 \\
4 & 1.15 & 0.55 & 39.70 \\
5 & 1.45 & 0.60 & 36.60 \\
6 & 1.65 & 0.30 & 46.25 \\
7 & 3.00 & 0.50 & 43.25 \\
\hline
\end{tabular}

Tabla 2. Valor medio de la concentración de cada una de las sustancias presentes en los anillos analizados. $\mathrm{Lec}=$ Acido lecanórico $; \mathrm{SNI}=$ Sustancia no identificada; $\mathrm{Gyr}=$ Acido girofórico .

La parte periférica del talo, es decir, la zona más alejada del ombligo, parece ser la que mantiene una mayor acumulación de sustancias liquénicas. Se trataría de la parte del liquen con un crecimiento más activo, a partir de la cual se produce el aumento en superficie del talo. Esto estaría en concordancia con el hecho de que a medida que el liquen aumenta en tamaño, edad, la proporción de sustancias por unidad de peso va disminuyendo.

El significado de estas variaciones, tanto las intratalinas como las que se aprecian en los talos de diferentes edades, permanece sin aclarar. Se necesitarían estudios fisiológicos de síntesis y acumulación de sustancias liquénicas « in vitro « que se pudieran correlacionar con variables de crecimiento real o de actividad. También incluso, pudiera tratarse de un fenómeno independiente de la propia actividad del liquen. El efecto de lavado del agua de lluvia podría ser más intenso en los talos con mayor superficie que en los más pequeños, en los que la relación peso-superficie es mucho más equilibrada.

\section{AGRADECIMIENTOS}

El pręsente trabajo ha sido realizado en el marco de los Proyectos de Investigación subvencionados por la CAICYT, número 2954-83 y DGICYT, número PB87-0229.

\section{BIBLIOGRAFÍA}

CULBERSON, C.F. - 1972- Improved conditions and new data for the identification of lichen products by a standardized thin-layer chromatographic method. J. Chromatogr. 92: 113125 .

CULBERSON, C.F. -1974- Conditions for the use of Merck silica gel 60 F254 plates in the standardized thin-layer chromatographic technique for lichen products. J. Chromatogr.97: 107-108. 
CULBERSON, C.F.\& W.L.CULBERSON -1958- Age and chemical constituents of individuals of the lichen Lasallia papulosa. Lloidia 21 (3): 189-192.

CULBERSON, C.F. \& H. KRISTINSSON -1970- A standardized method for the identification of lichen products. J. Chromatogr. 46: 85-93.

FAHSELT, D. -1984- Interthalline variability in levels of lichen products within stands of Cladina stellaris. Bryologist 87 (1): 50-56.

GEYER, M. -1985- Hochdruck-Flüssigkeits-Chromatographie (HPLC) von FlechtenSekundärstoffen. 233 pp. Diss. Univ. Essen. Essen.

LÓPEZ REDONDO, F. -1985-Aportaciones al conocimiento fitoquímico de Pseudevernia furfuracea (L.) Zopf en España. Universidad Complutense de Madrid. Memoria de Licenciatura inédita.

(Aceptado para su publicación en Junio de 1.990)

Dirección de los autores: E. Seriñá \& R. Arroyo: Dpto. de Biología Vegetal I, Facultad de Biología, Universidad Complutense, 28040 Madrid. E. Manrique: Dpto. de Biología Vegetal II, Facultad de Farmacia, Universidad Complutense, 28040 Madrid. 